\title{
Algebraically special resonances of the Kerr-black-hole-mirror bomb
}

\author{
Shahar Hod \\ The Ruppin Academic Center, Emeq Hefer 40250, Israel \\ and \\ The Hadassah Institute, Jerusalem 91010, Israel
}

(Dated: July 6, 2018)

\begin{abstract}
A co-rotating bosonic field interacting with a spinning Kerr black hole can extract rotational energy and angular momentum from the hole. This intriguing phenomenon is known as superradiant scattering. As pointed out by Press and Teukolsky, the black-hole-field system can be made unstable (explosive) by placing a reflecting mirror around the black hole which prevents the extracted energy from escaping to infinity. This composed black-hole-mirror-field bomb has been studied extensively by many researchers. It is worth noting, however, that most former studies of the black-hole bomb phenomenon have focused on the specific case of confined scalar (spin-0) fields. In the present study we explore the physical properties of the higher-spin (electromagnetic and gravitational) black-hole bombs. It is shown that this composed system is amenable to an analytic treatment in the physically interesting regime of rapidly-rotating black holes. In particular, we prove that the composed black-hole-mirror-field bomb is characterized by the unstable resonance frequency $\omega=m \Omega_{\mathrm{H}}+i s \cdot 2 \pi T_{\mathrm{BH}}$ (here $s$ and $m$ are respectively the spin-parameter and the azimuthal harmonic index of the field, and $\Omega_{\mathrm{H}}$ and $T_{\mathrm{BH}}$ are respectively the angular-velocity and the temperature of the rapidly-spinning black hole). Our results provide evidence that the higher-spin (electromagnetic and gravitational) black-hole-mirror bombs are much more explosive than the extensively studied scalar black-hole-mirror bomb. In particular, it is shown here that the instability growth rates which characterize the higher-spin black-hole bombs are two orders of magnitudes larger than the instability growth rate of the scalar black-hole bomb.
\end{abstract}




\section{INTRODUCTION}

Recent astrophysical observations provide compelling evidence that rapidly-rotating Kerr black holes are ubiquitous in our Universe [1-4]. These spinning black holes contain large amounts of rotational energy which can be released in various astrophysical processes. The mass-energy $M$ of a spinning Kerr black hole can be expressed in the form [5- 7]

$$
M=\sqrt{\frac{A}{16 \pi}+\frac{4 \pi J^{2}}{A}},
$$

where $J$ and $A$ are the angular-momentum and surface area of the black hole, respectively. Remembering that the black-hole surface area is an irreducible quantity in classical general relativity [8], one concludes that a fraction $1-1 / \sqrt{2}(\sim 29 \%)$ of the mass-energy of a maximally spinning Kerr black hole [9] is in the form of rotational energy. This is the fraction of energy which in principle can be extracted from a maximally spinning (extremal) Kerr black hole [10].

A concrete physical mechanism to extract energy from spinning black holes was suggested by Zel'dovich more than four decades ago [11]. The extraction mechanism is based on the remarkable phenomenon of superradiant amplification: co-rotating bosonic fields interacting with a spinning black hole [12 14] can gain energy (be amplified) on the expense of the black-hole rotational energy (this process is also characterized by the extraction of angular momentum from the spinning black hole).

Soon after the discovery of Zel'dovich [11] regarding the superradiant amplification of bosonic fields by spinning black holes, it was pointed out by Press and Teukolsky [15] that this remarkable mechanism can be used in order to build a powerful black-hole bomb. This bomb is made of three ingredients [15]: (1) a spinning black hole, (2) a bosonic field which interacts with the black hole to extract its rotational energy, and (3) a reflecting mirror which surrounds the black hole and prevents the extracted energy from escaping to infinity [16].

It was suggested in [15] that, the combined effect of superradiant amplification of the scattered bosonic field by the spinning black hole and its subsequent confinement by the reflecting mirror, would lead to an exponential growth in the field's amplitude (an exponential growth in the amount of rotational energy extracted from the central black hole). This

expectation was confirmed in the important work of Cardoso et. al. [17] for the specific case 
of confined scalar (spin-0) fields. In particular, it was established in [17] that, for a scalar mode of the form $e^{i m \phi-i \omega t}[18]$, the critical field frequency

$$
\omega=m \Omega_{\mathrm{H}}
$$

marks the onset of instability in the black-hole-mirror-scalar-field system. (Here $\Omega_{\mathrm{H}}$ is the angular-velocity of the black-hole horizon. This angular velocity can be expressed in the form $\Omega_{\mathrm{H}}=a / 2 M r_{+}$, where $a \equiv J / M$ and $r_{+}$are respectively the specific angular momentum and horizon-radius of the black hole).

Specifically, it was shown in [17] that, for confined scalar (spin-0) fields, the critical resonance frequency (2) sits on the boundary between stable and unstable black-hole-mirrorscalar-field configurations: confined scalar modes with $\Re \omega>m \Omega_{\mathrm{H}}$ decay in time (these scalar modes are characterized by $\Im \omega<0$ ) whereas confined scalar modes with $\Re \omega<m \Omega_{\mathrm{H}}$ grow exponentially over time (these scalar modes are characterized by $\Im \omega>0$ ).

In addition, using numerical techniques it was established in [17] that the instability growth rate [19] of a (confined and amplified) scalar field increases with increasing values of the black-hole spin parameter $a$. In other words, it was shown [17] that rapidly-rotating Kerr black holes are characterized by $\Im \omega$ values which are larger than the corresponding $\Im \omega$ values of slowly-rotating black holes. In particular, the numerical results of [17] reveal that the maximum instability growth rate of the black-hole-mirror-scalar-field bomb is characterized by [20]

$$
\Im \omega_{\max }^{\text {scalar }} \simeq 6 \times 10^{-5} M^{-1}
$$

It is worth emphasizing that this maximal value of $\Im \omega^{\text {scalar }}$ corresponds to confined scalar fields which are amplified by a maximally-spinning Kerr black hole.

It is worth emphasizing that most former studies of the black-hole bomb phenomenon have focused on the specific model of confined scalar (spin-0) fields. To the best of our knowledge, no analogous results exist in the literature for the physically interesting cases of confined higher-spin (electromagnetic and gravitational) fields. While the well-explored scalar black-hole-mirror bomb may serve as a simple toy model for the energy extraction mechanism from black holes, it should be realized that astrophysically realistic black holes are expected to be surrounded by conducting plasmas which may confine and reflect the electromagnetic (spin-1) perturbations modes. Likewise, deviations of the perturbed black 
hole from spherical symmetry are expected to produce gravitational (spin-2) wave modes which propagate in the black-hole spacetime.

In the present paper we shall use analytical techniques in order to explore the physical properties of these higher-spin black-hole-mirror bombs. In particular, the aim of the present paper is twofold:

(1) As emphasized above, it has been established [17] that the frequency $\Re \omega=m \Omega_{\mathrm{H}}$ corresponds to a marginally stable stationary resonance (with $\Im \omega=0$ ) of the scalar black-holemirror bomb. In the present paper we shall point out the (seemingly unknown) fact that, for confined higher-spin fields, the frequency $\Re \omega=m \Omega_{\mathrm{H}}$ may correspond to non-stationary unstable modes (with $\Im \omega>0$ ) of the electromagnetic and gravitational black-hole-mirror bombs.

(2) The second goal is to prove analytically that the instability growth rates (the values of $\Im \omega)$ which characterize the confined higher-spin fields are much larger than the instability growth rate (3) reported for the specific case of confined scalar (spin-0) fields. This fact, to be proved below, suggests that the higher-spin (electromagnetic and gravitational) blackhole-mirror bombs are much more explosive than the original black-hole-mirror-scalar-field bomb studied in [17].

\section{DESCRIPTION OF THE SYSTEM}

The physical system we consider consists of a massless bosonic field $\Psi$ linearly coupled to a spinning Kerr black hole of mass $M$ and angular momentum $M a$. In the Boyer-Lindquist coordinate system $(t, r, \theta, \phi)$ the black-hole spacetime is described by the line-element [21, 22]

$d s^{2}=-\left(1-\frac{2 M r}{\rho^{2}}\right) d t^{2}-\frac{4 M a r \sin ^{2} \theta}{\rho^{2}} d t d \phi+\frac{\rho^{2}}{\Delta} d r^{2}+\rho^{2} d \theta^{2}+\left(r^{2}+a^{2}+\frac{2 M a^{2} r \sin ^{2} \theta}{\rho^{2}}\right) \sin ^{2} \theta d \phi^{2}(4)$

where $\Delta \equiv r^{2}-2 M r+a^{2}$ and $\rho^{2} \equiv r^{2}+a^{2} \cos ^{2} \theta$. The zeroes of $\Delta$ :

$$
r_{ \pm}=M \pm\left(M^{2}-a^{2}\right)^{1 / 2}
$$

are the black-hole (event and inner) horizons. In order to facilitate a fully analytical study, we shall assume that the black hole is rapidly-rotating with $a \simeq M$. As shown in [17], the instability growth rate characterizing the composed black-hole-mirror bomb is an increasing function of the black-hole rotation parameter $a$. Hence, these rapidly-rotating 
(near-extremal) black holes are expected to produce the largest instability growth rates (the largest $\Im \omega$ values).

Teukolsky [23] has shown that the dynamics of massless scalar, electromagnetic, and gravitational perturbation fields in the rotating Kerr black-hole spacetime are governed by the single master equation [24]:

$$
\begin{array}{r}
{\left[\frac{\left(r^{2}+a^{2}\right)^{2}}{\Delta}-a^{2} \sin ^{2} \theta\right] \frac{\partial^{2} \Psi}{\partial t^{2}}+\frac{4 M a r}{\Delta} \frac{\partial^{2} \Psi}{\partial t \partial \phi}+\left(\frac{a^{2}}{\Delta}-\frac{1}{\sin ^{2} \theta}\right) \frac{\partial^{2} \Psi}{\partial \phi^{2}}} \\
-\Delta^{-s} \frac{\partial}{\partial r}\left(\Delta^{s+1} \frac{\partial \Psi}{\partial r}\right)-\frac{1}{\sin \theta} \frac{\partial}{\partial \theta}\left(\sin \theta \frac{\partial \Psi}{\partial \theta}\right)-2 s\left[\frac{a(r-M)}{\Delta}+\frac{i \cos \theta}{\sin ^{2} \theta}\right] \frac{\partial \Psi}{\partial \phi} \\
-2 s\left[\frac{M\left(r^{2}-a^{2}\right)}{\Delta}-r-i a \cos \theta\right] \frac{\partial \Psi}{\partial t}+\left(s^{2} \cot ^{2} \theta-s\right) \Psi=0 .
\end{array}
$$

Following [25] we shall use the "ingoing Kerr" coordinate system $(v, r, \theta, \bar{\phi})$ which is well behaved on the black-hole event horizon. These coordinates are related to the BoyerLindquist coordinates by the transformation equations [25]

$$
d v=d t+\left(r^{2}+a^{2}\right) d r / \Delta \text { and } \quad d \bar{\phi}=d \phi+a d r / \Delta
$$

Decomposing the field $\Psi$ in the form [26]

$$
{ }_{s} \Psi_{l m}=\sum_{l, m} e^{i m \bar{\phi}} S_{l m}(\theta ; a \omega){ }_{s} R_{l m}(r ; a, \omega) e^{-i \omega v}
$$

one finds [23] that the radial function ${ }_{s} R_{l m}$ and the angular function ${ }_{s} S_{l m}$ obey differential equations of the confluent Heun type [25, 27-29], see Eqs. (9) and (11) below.

It is worth emphasizing that the stability (or instability) of each mode is determined by the sign of $\Im \omega$ : stable (decaying in time) modes are characterized by $\Im \omega<0$ whereas unstable (growing in time) modes are characterized by $\Im \omega>0$. Stationary (marginally stable) solutions are characterized by $\Im \omega=0$.

The angular functions ${ }_{s} S_{l m}(\theta ; a \omega)$ are known as the spin-weighted spheroidal harmonics. These functions are solutions of the angular equation [25, 27, 29, 30]

$$
\frac{1}{\sin \theta} \frac{\partial}{\partial \theta}\left(\sin \theta \frac{\partial_{s} S_{l m}}{\partial \theta}\right)+\left[a^{2} \omega^{2} \cos ^{2} \theta-2 a \omega s \cos \theta-\frac{(m+s \cos \theta)^{2}}{\sin ^{2} \theta}-s^{2}+{ }_{s} E_{l m}\right]{ }_{s} S_{l m}=0
$$

in the interval $\theta \in[0, \pi]$. The boundary conditions of regularity at the poles $\theta=0$ and $\theta=\pi$ pick out a discrete set of eigenvalues ${ }_{s} E_{l m}(a \omega)$ labeled by the integers $m$ and $l \geq$ $\max \{|m|,|s|\}$. These angular eigenvalues can be expanded in the form [31, 32]

$$
{ }_{s} E_{l m}(a \omega)=l(l+1)+\sum_{k=1}^{\infty} c_{k}(a \omega)^{k}
$$


where the expansion coefficients $\left\{c_{k}(s, l, m)\right\}$ are given in [31, 32].

The radial function ${ }_{s} R_{l m}(r ; a, \omega)$ satisfies the differential equation [25]

$$
\Delta \frac{d^{2}{ }_{s} R_{l m}}{d r^{2}}+2[(1+s)(r-M)-i K] \frac{d_{s} R_{l m}}{d r}-\left[2(1+2 s) i \omega r+{ }_{s} \lambda_{l m}\right]_{s} R_{l m}=0,
$$

where $K \equiv\left(r^{2}+a^{2}\right) \omega-m a$ and

$$
{ }_{s} \lambda_{l m} \equiv{ }_{s} E_{l m}-2 m a \omega+a^{2} \omega^{2}-s(s+1) .
$$

Note that the angular equation (9) and radial equation (11) are coupled by the angular eigenvalues $\left\{{ }_{s} E_{l m}(a \omega)\right\}$. (We shall henceforth omit the indexes $s, l$ and $m$ for brevity.)

It is convenient to define new dimensionless variables

$$
x \equiv \frac{r-r_{+}}{r_{+}} ; \tau \equiv 8 \pi M T_{\mathrm{BH}}=\frac{r_{+}-r_{-}}{r_{+}} ; k=2 \omega r_{+} ; \varpi \equiv 4 M\left(\omega-m \Omega_{\mathrm{H}}\right),
$$

in terms of which the radial wave equation (111) becomes

$$
x(x+\tau) \frac{d^{2} R}{d x^{2}}-\left\{i k x^{2}+x[2 i k-2(1+s)]-(1+s) \tau+i \varpi\right\} \frac{d R}{d x}-[\lambda+i k(1+2 s)(x+1)] R=0 .
$$

We are interested in solutions of the radial wave equation (14) with the physical boundary condition of purely ingoing waves (as measured by a comoving observer) crossing the black-hole horizon [25]. As shown in [25], this physically motivated boundary condition corresponds to the behavior

$$
R\left(x \rightarrow x_{+}\right) \rightarrow 1
$$

of the radial function at the black-hole horizon. In addition, following [14, 17] we shall assume that the field vanishes at the location $x_{\mathrm{m}}$ of the mirror [33]:

$$
R\left(x=x_{\mathrm{m}}\right)=0
$$

The boundary conditions (15)-(16) single out a discrete family of complex frequencies (labeled by the integer resonance-parameter $n$ ). These characteristic frequencies are known as Boxed Quasi-Normal resonances $\left\{\omega^{\mathrm{BQN}}\left(n ; x_{\mathrm{m}}, M, a, s, l, m\right)\right\}[17,34]$.

\section{THE ALGEBRAICALLY SPECIAL RESONANCES OF THE BLACK-HOLE- MIRROR SYSTEM}

As we shall now show, the superradiant instability of the composed black-hole-mirrorfield system can be studied analytically in the regime of rapidly-rotating (near-extremal, 
$\tau \ll 1$ ) black holes enclosed in reflecting cavities which are placed in the near-horizon region $x_{\mathrm{m}} \ll 1$. In particular, we shall assume the following inequalities [35]:

$$
\max (\tau, \varpi) \ll x_{\mathrm{m}} \ll 1
$$

In the near-horizon region $x \ll 1$ [see Eq. (17)] the radial equation (14) can be approximated by

$$
x(x+\tau) \frac{d^{2} R}{d x^{2}}-\{x[2 i k-2(1+s)]-(1+s) \tau+i \varpi\} \frac{d R}{d x}-[\lambda+i k(1+2 s)] R=0 .
$$

The solution of equation (18) obeying the ingoing boundary condition (15) at the black-hole horizon is given by [25, 36]

$$
R(x)={ }_{2} F_{1}(s+1 / 2-i k+i \delta, s+1 / 2-i k-i \delta ; s+1-i \varpi / \tau ;-x / \tau),
$$

where ${ }_{2} F_{1}(a, b ; c ; z)$ is the hypergeometric function [36] and

$$
\delta^{2} \equiv k^{2}-\left(s+\frac{1}{2}\right)^{2}-\lambda .
$$

In the $x / \tau \gg \max (1, \varpi / \tau)$ regime [see Eq. (17)] one can use the large- $z$ asymptotic behavior of the hypergeometric function ${ }_{2} F_{1}(a, b ; c ; z)[36]$ to approximate the radial function (19) by [25, 37]

$$
R(x)=\frac{\Gamma(s+1-i \varpi / \tau) \Gamma(2 i \delta)}{\Gamma(s+1 / 2-i k+i \delta) \Gamma(1 / 2+i k+i \delta-i \varpi / \tau)}\left(\frac{x}{\tau}\right)^{-(s+1 / 2-i k-i \delta)}+(\delta \rightarrow-\delta) .
$$

The notation $(\delta \rightarrow-\delta)$ means "replace $\delta$ by $-\delta$ in the preceding term." We shall henceforth consider the case of real $\delta$ [38]. Using the expression (21) for the radial wave function, one can express the mirror-like boundary condition $R\left(x=x_{\mathrm{m}}\right)=0$ [see Eq. (16)] in the form

$$
\left(\frac{x_{\mathrm{m}}}{\tau}\right)^{2 i \delta} \frac{\Gamma(2 i \delta) \Gamma(s+1 / 2-i k-i \delta) \Gamma(1 / 2+i k-i \delta-i \varpi / \tau)}{\Gamma(-2 i \delta) \Gamma(s+1 / 2-i k+i \delta) \Gamma(1 / 2+i k+i \delta-i \varpi / \tau)}=-1 .
$$

As emphasized above, it has been established in [17] that, for confined scalar $s=0$ fields, the resonance frequency $\Re \omega=m \Omega_{\mathrm{H}}$ [see Eq. (2)] corresponds to a marginally stable mode with $\Im \omega=0$. This frequency therefore marks the boundary between stable $(\Im \omega<0)$ and unstable $(\Im \omega>0)$ black-hole-mirror-scalar-field configurations [17].

However, a close inspection of the resonance condition (22) reveals the somewhat surprising fact that, for confined higher-spin $s \neq 0$ fields, the frequency $\Re \omega=m \Omega_{\mathrm{H}}$ may 
correspond to a non-stationary mode with $\Im \omega \neq 0$. In particular, we shall now prove that the algebraically special [39] complex frequency

$$
\omega=m \Omega_{\mathrm{H}}+i s \times \frac{\tau}{4 M}
$$

is a valid solution of the resonance equation (22) for electromagnetic $(s=1)$ and gravitational $(s=2)$ fields [40]. Note that the resonance (23) is characterized by $\Im \omega>0$. This resonance frequency thus corresponds to an unstable mode of the Kerr-black-hole-mirror-field system.

This property of higher-spin fields can be verified directly by substituting $\varpi=i s \times \tau$ [40] into the resonance condition (22) and taking the logarithm of both sides of this equation. One then finds the characteristic equation [41]

$2 \delta \ln \left(\frac{x_{\mathrm{m}}}{\tau}\right)=i \ln \left[\frac{\Gamma(2 i \delta)}{\Gamma(-2 i \delta)}\right]+i \ln \left[\frac{\Gamma(s+1 / 2-i k-i \delta)}{\Gamma(s+1 / 2+i k+i \delta)}\right]+i \ln \left[\frac{\Gamma(s+1 / 2+i k-i \delta)}{\Gamma(s+1 / 2-i k+i \delta)}\right]+\pi(2 n+1)$

for the dimensionless radii $\left\{x_{\mathrm{m}}\right\}$ of the mirror, where the resonance parameter $n$ is an integer.

Inspection of the resonance equation (24) reveals the following facts: the first three terms on the r.h.s of this equation are of the form $i[\ln (z)-\ln (\bar{z})]$ and are therefore purely real [42]. The fourth term on the r.h.s of (24) is obviously a purely real number. One therefore concludes that the r.h.s of (24) is purely real. Hence, the l.h.s of (24) must also be a purely real number. This implies that, for real values of $\delta$, the solutions to the characteristic equation (24):

$$
x_{\mathrm{m}}(n)=x_{\mathrm{m}}(n=0) \times e^{\pi n / \delta}
$$

with

$$
x_{\mathrm{m}}(0)=\tau \times\left[\frac{\Gamma(-2 i \delta) \Gamma(s+1 / 2+i k+i \delta) \Gamma(s+1 / 2-i k+i \delta)}{\Gamma(2 i \delta) \Gamma(s+1 / 2-i k-i \delta) \Gamma(s+1 / 2+i k-i \delta)}\right]^{1 / 2 i \delta} e^{\pi / 2 \delta}
$$

are real numbers [otherwise, the l.h.s of (24) would not be a purely real number].

The discrete radii (25)-(26) correspond to the possible radial locations of the mirror with the algebraically special resonance frequency (23). In Tables \and [1] we display these characteristic mirror radii for equatorial $l=m$ [43] electromagnetic $(s=1)$ and gravitational $(s=2)$ modes [44]. It is worth emphasizing again that our analysis is valid in the regime $\tau \ll x_{\mathrm{m}}$ [see Eq. (17)]. Thus, in Tables [1] and [1] we present results for mirror radii with the property $x_{\mathrm{m}} / \tau>10[45]$. 
From Tables \ and $\llbracket$ one learns that the radii of the mirror $\left\{x_{\mathrm{m}}(n ; s, m)\right\}$ increase with increasing values of the overtone number $n$ [see also Eq. (25)] and decrease with increasing values of the azimuthal harmonic index $m$. It is worth emphasizing again that these mirror radii correspond to the algebraically special unstable resonance frequency (23).

\begin{tabular}{|c|c|c|c|c|c|}
\hline$l=m$ & $\delta$ & $x_{\mathrm{m}}(n=1) / \tau$ & $x_{\mathrm{m}}(n=2) / \tau$ & $x_{\mathrm{m}}(n=3) / \tau$ & $x_{\mathrm{m}}(n=4) / \tau$ \\
\hline 1 & 0.492 & 2225.86 & $1.33 \times 10^{6}$ & $7.89 \times 10^{8}$ & $4.70 \times 10^{11}$ \\
2 & 1.336 & 28.80 & 302.36 & 3174.61 & 33331.40 \\
3 & 2.198 & - & 41.03 & 171.32 & 715.39 \\
4 & 3.055 & - & 17.06 & 47.71 & 133.43 \\
\hline
\end{tabular}

TABLE I: The scaled mirror radii $x_{\mathrm{m}} / \tau$ which correspond to the equatorial $l=m$ electromagnetic $s=1$ modes with the algebraically special resonance frequency (23). We display results for the resonances $n=1$ to $n=4$ with the property $x_{\mathrm{m}}(n) / \tau>10$. Also shown are the corresponding values of the parameter $\delta$ [see Eq. (20)]. One finds that the radius of the mirror $x_{\mathrm{m}}(n ; s=1, m)$ is an increasing function of the overtone number $n$ and a decreasing function of the azimuthal harmonic index $m$.

\begin{tabular}{|c|c|c|c|c|c|}
\hline$l=m$ & $\delta$ & $x_{\mathrm{m}}(n=1) / \tau$ & $x_{\mathrm{m}}(n=2) / \tau$ & $x_{\mathrm{m}}(n=3) / \tau$ & $x_{\mathrm{m}}(n=4) / \tau$ \\
\hline 2 & 2.051 & 11.54 & 53.37 & 246.91 & 1142.36 \\
3 & 2.794 & - & 21.23 & 65.36 & 201.24 \\
4 & 3.565 & - & 12.33 & 29.76 & 71.83 \\
5 & 4.358 & - & - & 17.75 & 36.51 \\
\hline
\end{tabular}

TABLE II: The scaled mirror radii $x_{\mathrm{m}} / \tau$ which correspond to the equatorial $l=m$ gravitational $s=2$ modes with the algebraically special resonance frequency (23). We display results for the resonances $n=1$ to $n=4$ with the property $x_{\mathrm{m}}(n) / \tau>10$. Also shown are the corresponding values of the parameter $\delta$ [see Eq. (20)]. One finds that the radius of the mirror $x_{\mathrm{m}}(n ; s=2, m)$ is an increasing function of the overtone number $n$ and a decreasing function of the azimuthal harmonic index $m$.

In order to check the accuracy of our analytical results, we have solved Eq. (19) numerically with the mirror boundary condition (16). For the fundamental gravitational mode 
with $l=m=2$ we find that the exact (numerical) result agrees with our analytical result [see Eq. (26)] to within a small correction factor of the order of $\sim 1.4 \tau / x_{\mathrm{m}} \ll 1$ [46].

\section{SUMMARY AND DISCUSSION}

We have used analytical tools in order to explore the superradiant instability phenomenon of rapidly-spinning Kerr black holes enclosed inside reflecting cavities (the composed blackhole-mirror bomb). Imposing a mirror-like boundary condition on co-rotating higher-spin (electromagnetic and gravitational) fields in the vicinity of the black-hole horizon, it was shown that the confined bosonic fields grow exponentially over time.

While most former studies of the black-hole-bomb phenomenon have considered the specific case of confined scalar $(s=0)$ fields, in the present study we have focused on the physically interesting cases of confined electromagnetic $(s=1)$ and gravitational $(s=2)$ fields.

Former studies [17] of the black-hole-mirror-scalar-field system have established that the critical frequency $\Re \omega=m \Omega_{\mathrm{H}}$ [see Eq. (2)] marks the onset of instability in this composed system [47] (that is, $\Re \omega=m \Omega_{\mathrm{H}}$ corresponds to a stationary scalar mode with $\Im \omega=0$ ). On the other hand, in the present study we have revealed the (somewhat surprising) fact that this property is actually not a generic feature of composed black-hole-mirror bombs. In particular, we have shown that, for confined higher-spin $s \neq 0$ fields, the frequency $\Re \omega=m \Omega_{\mathrm{H}}$ may correspond to a non-stationary mode with $\Im \omega \neq 0$.

Although the equations governing the dynamics of the confined fields in the black-hole spacetime are rather complex [see, in particular, the coupled equations (9), (12), and (14)], we have succeeded to prove analytically that the algebraically special [39] frequency $\omega=$ $m \Omega_{\mathrm{H}}+s \cdot i 2 \pi T_{\mathrm{BH}}$ [see Eq. (23)] is a valid solution of the resonance condition (22). That is, we have shown that this complex frequency corresponds to an unstable resonance of the composed black-hole-mirror-field system [48].

It is worth emphasizing that our analysis is valid in the regime of rapidly-rotating (nearextremal) black holes with $\tau \ll x_{\mathrm{m}} \ll 1$ [see Eq. (17)]. Specifically, since each inequality sign in (17) roughly corresponds to an order-of-magnitude difference between two variables (that

is, $\tau / x_{\mathrm{m}} \lesssim 10^{-1}$ with $x_{\mathrm{m}} \lesssim 10^{-1}$ ), our results should be valid in the regime $\tau \lesssim 10^{-3}$ [49]. Thus, the algebraically special resonance (23) for confined electromagnetic and gravitational 
fields is characterized by

$$
\Im \omega_{\max }=O\left(10^{-3} M^{-1}\right)
$$

The present analysis provides compelling evidence that the higher-spin (electromagnetic and gravitational) black-hole-mirror bombs are much more explosive than the original blackhole-mirror-scalar-field bomb studied in [17]. In particular, taking cognizance of Eqs. (33) and (27), one concludes that the instability growth rates (the values of $\Im \omega$ ) which characterize

the superradiant confined higher-spin $s \neq 0$ fields are much larger than the maximal growth rate (3) which characterizes a superradiant confined scalar $s=0$ field [50].

Finally, it is worth emphasizing that the present analytical exploration of the black-holebomb phenomenon is restricted to the regime of linear perturbation fields. As we have seen, the initial exponential growth of the confined fields (the superradiant instability) manifests itself at this linear level. However, it is clear that a fully non-linear numerical simulation of the coupled black-hole-field dynamics is required in order to reveal the end-state of this explosive superradiant instability.

\section{ACKNOWLEDGMENTS}

This research is supported by the Carmel Science Foundation. I thank Yael Oren, Arbel M. Ongo and Ayelet B. Lata for helpful discussions.

[1] E. Berti and M. Volonteri, Astrophys. J. 684, 822 (2008).

[2] L. Brenneman, C. Reynolds, M. Nowak, R. Reis, M. Trippe, et al., Astrophys.J. 736, 103 (2011).

[3] C. S. Reynolds, e-print arXiv:1307.3246.

[4] H. Yang, A. Zimmerman, A. Zenginoglu, F. Zhang, E. Berti, and Y. Chen, Phys. Rev. D 88, 044047 (2013).

[5] D. Christodoulou, Phys. Rev. Lett. 25, 1596 (1970).

[6] D. Christodoulou and R. Ruffni, Phys. Rev. D 4, 3552 (1971).

[7] We use natural units in which $G=c=\hbar=1$.

[8] S. W. Hawking, Phys. Rev. Lett. 26, 1344 (1971).

[9] An extremal (maximally spinning) Kerr black hole is characterized by $J=M^{2}=A / 8 \pi$. 
[10] This maximal extraction of energy from a spinning black hole can be achieved in a reversible process in which an extremal (maximally spinning) Kerr black hole of mass $M$, surface area $A=8 \pi M^{2}$, and angular-momentum $J=M^{2}$ is transformed into a non-spinning $J=0$ Schwarzschild black hole with the same surface area $A$ and mass $M / \sqrt{2}$.

[11] Ya. B. Zel'dovich, Pis'ma Zh. Eksp. Teor. Fiz. 14, 270 (1971) [JETP Lett. 14, 180 (1971)]; Zh. Eksp. Teor. Fiz. 62, 2076 (1972) [Sov. Phys. JETP 35, 1085 (1972)]; A. V. Vilenkin, Phys. Lett. B 78, 301 (1978).

[12] An analogous extraction of Coulomb energy may occur when charged scalar fields interact with charged Reissner-Nordström black holes [13, 14].

[13] J. D. Bekenstein, Phys. Rev. D 7, 949 (1973).

[14] J. C. Degollado, C. A. R. Herdeiro, and H. F. Rúnarsson, Phys. Rev. D 88, 063003 (2013); S. Hod, Phys. Rev. D 88, 064055 (2013).

[15] W. H. Press and S. A. Teukolsky, Nature 238, 211 (1972); W. H. Press and S. A. Teukolsky, Astrophys. J. 185, 649 (1973).

[16] It was later suggested to replace the massless bosonic fields by massive ones. The mass term of a massive field may effectively act as a reflecting mirror. In this case a potential well which bounds the massive field to the central black hole may be formed in the black-hole exterior, thus preventing the extracted energy (the amplified field) from escaping to infinity. For this version of the black-hole bomb, see: T. Damour, N. Deruelle and R. Ruffini, Lett. Nuovo Cimento 15, 257 (1976); T. M. Zouros and D. M. Eardley, Annals of physics 118, 139 (1979); S. Detweiler, Phys. Rev. D 22, 2323 (1980); H. Furuhashi and Y. Nambu, Prog. Theor. Phys. 112, 983 (2004); V. Cardoso and J. P. S. Lemos, Phys. Lett. B 621, 219 (2005); V. Cardoso and S. Yoshida, JHEP 0507:009 (2005); S. R. Dolan, Phys. Rev. D 76, 084001 (2007); S. Hod and O. Hod, Phys. Rev. D 81, Rapid communication 061502 (2010) arXiv:0910.0734; H. R. Beyer, J. Math. Phys. 52, 102502 (2011); S. Hod, Phys. Lett. B 708, 320 (2012) arXiv:1205.1872]; S. Hod, Phys. Lett. B 713, 505 (2012); S. Hod, Phys. Rev. D 86, 104026 (2012) arXiv:1211.3202]; S. Hod, Phys. Lett. B 718, 1489 (2013); S. Hod, The Euro. Phys. Journal C 73, 2378 (2013); R. Brito, V. Cardoso, and P. Pani, Phys. Rev. D 88, 023514 (2013); S. R. Dolan, Phys. Rev. D 87, 124026 (2013); H. Witek, V. Cardoso, A. Ishibashi, and U. Sperhake, Phys. Rev. D 87, 043513 (2013); V. Cardoso, Gen. Relativ. and Gravit. 45, 2079 (2013). 
[17] V. Cardoso, O. J. C. Dias, J. P. S. Lemos and S. Yoshida, Phys. Rev. D 70, 044039 (2004); Erratum-ibid. D 70, 049903 (2004).

[18] Here $\omega$ and $m$ are respectively the conserved frequency and azimuthal harmonic index of the scalar wave field.

[19] The instability growth rate of the field is characterized by the imaginary part $\Im \omega$ of its frequency.

[20] Note that this (maximal) value of $\Im \omega^{\text {scalar }}$ corresponds to an instability timescale $T_{\text {ins }}^{\text {scalar }} \equiv$ $1 / \Im \omega^{\text {scalar }} \simeq \frac{1}{6} \times 10^{5} M$ which is much longer than the dynamical timescale set by the mass $M$ of the black hole.

[21] S. Chandrasekhar, The Mathematical Theory of Black Holes, (Oxford University Press, New York, 1983).

[22] R. P. Kerr, Phys. Rev. Lett. 11, 237 (1963).

[23] S. A. Teukolsky, Phys. Rev. Lett. 29, 1114 (1972); S. A. Teukolsky, Astrophys. J. 185, 635 (1973).

[24] The dependent variables in the black-hole perturbation equations are related to null tetrad components of the electromagnetic and gravitational (Weyl) fields, see 23] for details. The parameter $s$ in the Teukolsky master equation (6) is known as the spin-weight of the field. This parameter takes different values for different perturbation fields [23]. In particular, a scalar perturbation field $\phi$ is characterized by $s=0$; the electromagnetic perturbation field $\phi_{0}$ is characterized by $s=1$ and the electromagnetic perturbation field $(r-i a \cos \theta)^{2} \phi_{2}$ is characterized by $s=-1$; the gravitational perturbation field $\psi_{0}$ is characterized by $s=2$ and the gravitational perturbation field $(r-i a \cos \theta)^{4} \psi_{4}$ is characterized by $s=-2$. (see [23] for the precise definitions of the variables $\phi_{0}, \phi_{2}, \psi_{0}$, and $\left.\psi_{4}\right)$.

[25] S. A. Teukolsky and W. H. Press, Astrophys. J. 193, 443 (1974).

[26] Here $l$ and $m$ are respectively the spheroidal and azimuthal harmonic indexes of the mode [see Eq. (9) below], and $\omega$ is the conserved frequency of the wave field.

[27] C. Flammer, Spheroidal Wave Functions (Stanford University Press, Stanford, 1957).

[28] P. P. Fiziev, e-print arXiv:0902.1277; R. S. Borissov and P. P. Fiziev, e-print arXiv:0903.3617; P. P. Fiziev, Phys. Rev. D 80, 124001 (2009); P. P. Fiziev, Class. Quant. Grav. 27, 135001 (2010).

[29] A. Ronveaux, Heun's differential equations (Oxford University Press, Oxford, UK, 1995). 
[30] S. Hod, Phys. Lett. B 717, 462 (2012) arXiv:1304.0529]; S. Hod, Phys. Rev. D 87, 064017 (2013) arXiv:1304.4683.

[31] W. H. Press and S. A. Teukolsky, Astrophys. J. 185, 649 (1973).

[32] E. Berti, V. Cardoso, and M. Casals, Phys. Rev. D 73, 024013 (2006); Erratum-ibid. D 73, $109902(2006)$.

[33] Note that in the Boyer-Lindquist coordinate system one should replace the expansion (8) by ${ }_{s} \Psi_{l m}=\sum_{l, m} e^{i m \phi}{ }_{s} S_{l m}(\theta ; a \omega)_{s} R_{l m}^{\mathrm{BL}}(r ; a, \omega) e^{-i \omega t}$. The relation between the radial functions $R$ and $R^{\mathrm{BL}}$ is given by $R^{\mathrm{BL}}=R \cdot \exp \left[-i \int(K / \Delta) d r\right.$ ] [Here we have used the relations $v=$ $t+\int\left[\left(r^{2}+a^{2}\right) / \Delta\right] d r$ and $\bar{\phi}=\phi+\int(a / \Delta) d r$, see Eq. (7)]. Thus, the functions $R$ and $R^{\mathrm{BL}}$ share the same set of radial zeroes.

[34] Note that the extensively studied family of black-hole quasinormal resonances is characterized by the boundary condition of outgoing waves at spatial infinity [25, 31].

[35] Specifically, below we shall assume the inequalities $x_{\mathrm{m}}<10^{-1}$ and $\tau / x_{\mathrm{m}}<10^{-1}$.

[36] M. Abramowitz and I. A. Stegun, Handbook of Mathematical Functions (Dover Publications, New York, 1970).

[37] It is worth emphasizing that the expression (21) for the radial wave function $R(x)$ is valid in the regime $\tau \ll x \ll 1$, see Eq. (17).

[38] One can choose $\delta>0$ without loss of generality.

[39] We use the term "algebraically special" to emphasize the fact that this resonance frequency of the composed black-hole-mirror-field system can be identified analytically.

[40] Note that the resonance frequency (23) corresponds to $\varpi=i s \times \tau$ [see Eq. (13)]. In addition, note that the imaginary part of this unstable resonance can be expressed in terms of the black-hole temperature as $\Im \omega=s \cdot 2 \pi T_{\mathrm{BH}}$.

[41] Here we have used the relation $-1=e^{i \pi(2 n+1)}$ which implies $\ln (-1)=i \pi(2 n+1)$, where $n$ is an integer.

[42] See Eq. 6.1.23 of [36].

[43] It is well known that these equatorial $l=m$ modes are the most amplified ones [17, 25].

[44] We are interested here in the relation $x_{\mathrm{m}}=x_{\mathrm{m}}(\tau)$ to leading-order in the small parameter $\tau \ll 1$. We therefore take $a \omega=m / 2+O(\tau)$ and $k=m+O\left(\tau^{2}\right)$ in Eqs. (10), (12), (20), (25), and (26). The leading-order values of the constants ${ }_{s} \delta_{l m}$ are given in Tables $\Pi$ and II,

[45] We find that modes with $n \leq 0$ are characterized by $x_{\mathrm{m}} / \tau<10$. Hence, these modes are 
beyond the regime of validity of our analysis.

[46] That is, $x_{\mathrm{m}}^{\text {analytical }} / x_{\mathrm{m}}^{\text {numerical }} \simeq 1+1.4 \tau / x_{\mathrm{m}}^{\text {numerical }} \simeq 1$.

[47] As explained above, for the specific case of confined scalar $s=0$ fields, one finds [17] that scalar modes with $\Re \omega>m \Omega_{\mathrm{H}}$ are stable $(\Im \omega<0)$ whereas scalar modes with $\Re \omega<m \Omega_{\mathrm{H}}$ are unstable $(\Im \omega>0)$. The critical scalar mode with $\Re \omega=m \Omega_{\mathrm{H}}$ is marginally stable (stationary) with $\Im \omega=0$.

[48] The fact that, for confined higher-spin $s \neq 0$ fields, the mode with $\Re \omega=m \Omega_{\mathrm{H}}$ corresponds to an unstable resonance with $\Im \omega>0$, implies that the spectrum of unstable higher-spin resonances contains frequencies with $\Re \omega>m \Omega_{\mathrm{H}}$. This is to be contrasted with the scalar $s=0$ field case, in which the unstable resonances are characterized by $\Re \omega<m \Omega_{\mathrm{H}}$.

[49] It should be realized that here we have used a very conservative estimate for the regime of validity of our analysis. In fact, the two inequalities $\tau \ll x_{\mathrm{m}} \ll 1\left(\tau / x_{\mathrm{m}} \lesssim 10^{-1}\right.$ with $x_{\mathrm{m}} \lesssim 10^{-1}$ ) may be satisfied simultaneously in the less restrictive regime $\tau \lesssim 10^{-2}$.

[50] In particular, equations (3) and (27) imply that the electromagnetic and gravitational blackhole-mirror bombs are characterized by instability timescales which are at least two orders of magnitude shorter than the instability timescale of the scalar black-hole-mirror bomb. 